\title{
In Vivo Labeling of Serum Albumin for PET
}

\author{
Gang Niu*, Lixin Lang*, Dale O. Kiesewetter, Ying Ma, Zhongchan Sun, Ning Guo, Jinxia Guo, Chenxi Wu, \\ and Xiaoyuan Chen
}

Laboratory of Molecular Imaging and Nanomedicine (LOMIN), National Institute of Biomedical Imaging and Bioengineering (NIBIB), National Institutes of Health (NIH), Bethesda, Maryland

The purpose of this study was to develop a novel in vivo albuminlabeling method to allow PET of cardiac function after myocardial infarction and vascular leakage and increased permeability in inflammatory diseases and malignant tumors. Methods: To label albumin in vivo, we synthesized a NOTA (1,4,7-triazacyclononane$N, N^{\prime}, N^{\prime \prime}$-triacetic acid)-conjugated truncated form of Evans blue (NEB). ${ }^{18} \mathrm{~F}$ labeling was achieved by the formation of an ${ }^{18} \mathrm{~F}$-aluminum fluoride $\left({ }^{18} \mathrm{~F}-\mathrm{AIF}\right)$ complex, and ${ }^{64} \mathrm{Cu}$ labeling was obtained by a standard chelation method. Sixty-minute dynamic PET imaging was performed on normal mice to evaluate the distribution of ${ }^{18} \mathrm{~F}$-AIF-NEB, which was compared with in vitro-labeled mouse serum albumin ( ${ }^{18} \mathrm{~F}$-fluorobenzylMSA). Electrocardiography-gated PET imaging was performed in a mouse model of myocardial infarction. Both dynamic and static PET scans were obtained in a mouse inflammation model induced by local injection of turpentine to evaluate vascular leakage. Tumor permeability was studied by dynamic and late-point static PET using ${ }^{64} \mathrm{Cu}-\mathrm{NEB}$ in a UM-22B xenograft model. Results: NEB was successfully synthesized, and ${ }^{18} \mathrm{~F}$ labeling including work-up took about 20-30 min, with a radiochemical purity greater than $95 \%$ without the need for high-performance liquid chromatography purification. Most of the radioactivity was retained in the circulation system at $60 \mathrm{~min}$ after injection (26.35 \pm 1.52 percentage injected dose per gram [\%ID/g]). With electrocardiography-gated PET, ventricles of the heart and major arteries were clearly visualized. The myocardial infarction mice showed much lower left ventricular ejection fraction than the control mice. Inflammatory muscles showed significantly higher tracer accumulation than the contralateral healthy ones. UM-22B tumor uptake of ${ }^{64} \mathrm{Cu}-\mathrm{NEB}$ gradually increased with time $(5.73 \pm 1.11 \% \mathrm{ID} / \mathrm{g}$ at $1 \mathrm{~h}$ and $8.03 \pm 0.77 \% \mathrm{ID} / \mathrm{g}$ at $2 \mathrm{~h}$ after injection). Conclusion: The distribution and local accumulation of serum albumin can be noninvasively visualized and quantified by ${ }^{18} \mathrm{~F}-\mathrm{AIF}-\mathrm{NEB}$ and ${ }^{64} \mathrm{Cu}-\mathrm{NEB}$ PET. The simple labeling and broad applications make these imaging probes attractive for clinical translation.

Key Words: serum albumin; in vivo labeling; Evans blue; PET; vascular permeability

J Nucl Med 2014; 55:1150-1156

DOI: 10.2967/jnumed.114.139642

\footnotetext{
Received Feb. 24, 2014; revision accepted Mar. 24, 2014.

For correspondence or reprints contact: Xiaoyuan Chen, Laboratory of Molecular Imaging and Nanomedicine (LOMIN), Bldg. 35A, Rm. GD737, National Institute of Biomedical Imaging and Bioengineering (NIBIB), National Institutes of Health (NIH), Bethesda, MD 20892.

E-mail: shawn.chen@nih.gov

${ }^{*}$ Contributed equally to this work.

Published online May 19, 2014.

COPYRIGHT (c) 2014 by the Society of Nuclear Medicine and Molecular Imaging, Inc.
}

A the most abundant plasma protein, serum albumin has emerged as a versatile carrier for therapeutic agents, primarily for treating diabetes, cancer, rheumatoid arthritis, and infectious diseases (1). Serum albumin was also used directly as an imaging probe after labeling with fluorescent dyes (2) for optical imaging, radioisotopes for scintillation scanning or PET $(3,4)$, or $\mathrm{Gd}^{3+}$ for MR imaging (5). The major applications of labeled serum albumin mentioned include blood-pool imaging and angiography.

In clinical nuclear medicine, kit preparations for indirect and direct ${ }^{99 \mathrm{~m}} \mathrm{Tc}$ radiolabeling of red blood cells (RBCs) are still the dominant methods for blood-pool imaging. Compared with SPECT, PET is more sensitive and has higher spatial resolution with clinical instruments. However, to date, only very few blood-pool tracers have been introduced for PET. For example, carbon monoxide containing either ${ }^{11} \mathrm{C}$ or ${ }^{15} \mathrm{O}$ has been used to label $\mathrm{RBCs}$ for PET (6). However, because of their short half-lives (20.4 min for ${ }^{11} \mathrm{C}$ and 2.05 min for ${ }^{15} \mathrm{O}$ ), these tracers can be used only in centers with an in-house cyclotron. Moreover, the gaseous form of carbon monoxide and the need for administration by inhalation necessitates sophisticated equipment for either human or animal studies (7).

The commercial availability of the species-specific isoforms of albumin including human serum albumin makes blood drawing unnecessary. In fact, ${ }^{131}$ I-labeled human serum albumin is the only radiologic agent approved by the Food and Drug Administration for measuring blood volume. For imaging purposes, albumin has been labeled with various radioisotopes for PET imaging including ${ }^{68} \mathrm{Ga}$ (4), ${ }^{62} \mathrm{Cu}(8)$, and ${ }^{64} \mathrm{Cu}(9)$. Compared with these radiometals, ${ }^{18} \mathrm{~F}$ has the advantages of being a pure position emitter and having an ideal half-life. It is the dominant radioisotope used for PET imaging for both clinical applications and preclinical investigations.

As a protein, albumin can be labeled with ${ }^{18} \mathrm{~F}$ through reaction of $\mathrm{N}$-succinimidyl $4-{ }^{18} \mathrm{~F}$-fluorobenzoate $\left({ }^{18} \mathrm{~F}-\mathrm{SFB}\right)$ with an amine group or $N$-[2-(4- ${ }^{18} \mathrm{~F}$-fluorobenzamido)ethyl]maleimide on the thiol group (10). In 1 study, Wangler et al. (11) prepared 4-(di-tert-butyl- ${ }^{18} \mathrm{~F}-$ fluorosilyl)benzenethiol and coupled it directly to rat serum albumin (RSA). However, high liver uptake was observed on the ${ }^{18} \mathrm{~F}-\mathrm{SiFA}-$ RSA blood-pool scan, indicating that the albumin structure may have been disrupted to some extent during labeling. One alternative is in vivo labeling of endogenous albumin with a prelabeled albumin binder. Ideally, the binder will not affect the in vivo behavior of the serum albumin such as circulation, extravascularization, and turnover; thus, the imaging results will reflect the distribution and metabolism of serum albumin accurately. Currently available albumin binders include small molecules, peptides that possess an albumin binding domain, and antibodies (12).

Evans blue (EB) dye has been an important tool in many physiologic and clinical investigations because of its high affinity 
for serum albumin (13). EB has been used in clinical practice for almost $90 \mathrm{y}$ as a pharmaceutical product, principally for the determination of patient plasma volume (14). Although recently discontinued in clinical use, EB is still used as a sensitive and qualitative marker of leakage of protein from the vascular lumen in a variety of tissues subjected to inflammation and traumatic injury $(15,10)$. It has also served as a surrogate marker for serum albumin flux across the luminal barrier in many in vivo experimental situations $(17,18)$.

By taking advantage of the high in vivo binding affinity of EB to albumin, here, we developed a NOTA (1,4,7-triazacyclononane- $N$, $N^{\prime}, N^{\prime \prime}$-triacetic acid) conjugate of a truncated form of Evans blue (NEB) for in vivo albumin labeling. ${ }^{18} \mathrm{~F}$ labeling was achieved by the formation of ${ }^{18} \mathrm{~F}$-aluminum fluoride $\left({ }^{18} \mathrm{~F}-\mathrm{AlF}\right)$ complex $(19-21)$, and ${ }^{64} \mathrm{Cu}$ labeling was through standard chelation chemistry. Small-animal PET was performed with this novel tracer to evaluate cardiac function and enhanced vascular permeability in animal models with inflammatory diseases and tumors.

\section{MATERIALS AND METHODS}

\section{General Materials and Instrumentation}

Monosodium salt of 1-amino-8-naphthol-2, 4-disulfonic acid was purchased from TCI America, and all other chemicals were from SigmaAldrich. Mass spectra (MS) were obtained on a Waters Acquity UPLC system coupled with Waters Q-Tof Premier MS (liquid chromatographymass spectrometry [LC-MS]). Semipreparative reversed-phase highperformance liquid chromatography (HPLC) was performed on a Waters 600 gradient system with a Waters 996 Photodiode Array detector using a Waters Nova-Pak HR $\mathrm{C}_{18}$ column $(6 \mu \mathrm{m}, 300 \times 7.8 \mathrm{~mm})$. Analytic reversed-phase HPLC was performed on a Perkin-Elmer Series 200 LC gradient system with a Waters 2784 Dual Absorbance ultraviolet detector plus a Bioscan radioisotope detector using a Waters Symmetry column $(5 \mu \mathrm{m}, 150 \times 3.9 \mathrm{~mm})$. The flow rate was $6 \mathrm{~mL} / \mathrm{min}$ for the semipreparative column and $1 \mathrm{~mL} / \mathrm{min}$ for the analytic column running the same linear gradient starting from $5 \% \mathrm{~A}(0.1 \%$ trifluoroacetic acid in acetonitrile) and $95 \% \mathrm{~B}(0.1 \%$ trifluoroacetic acid in water) for $5 \mathrm{~min}$ and increasing $\mathrm{A}$ to $65 \%$ at $35 \mathrm{~min}$. A Varian Bond Elut $\mathrm{C}_{18}$ column $(100 \mathrm{mg})$ was used for solid-phase extraction of the labeled product. ${ }^{18} \mathrm{~F}$-fluoride and ${ }^{64} \mathrm{CuCl}_{2}$ were obtained from the National Institutes of Health cyclotron facility.

\section{Preparation of NOTA-Tolidine}

To a 4-mL glass vial containing $20.0 \mathrm{mg}$ of o-tolidine ( $94 \mu \mathrm{mol})$ and $20.0 \mathrm{mg}$ of NOTA-3HCl $(48 \mu \mathrm{mol})$ in $1 \mathrm{~mL}$ of dimethyl sulfoxide was added $3.6 \mu \mathrm{L}$ of diethyl cyanophosphonate $(24 \mu \mathrm{mol})$ and $25 \mu \mathrm{L}$ of diisopropylethylamine. The mixture was stirred at room temperature for $40 \mathrm{~min}$, and another $3.6 \mu \mathrm{L}$ of diethyl cyanophosphonate were added and stirred at room temperature overnight. The mixture was then purified with semipreparative HPLC. The peak containing the desired product was collected $\left(\mathrm{R}_{\mathrm{t}}=10.0 \mathrm{~min}\right)$, and the solution was frozen over dry ice and lyophilized overnight to give $12.2 \mathrm{mg}$ of pure product in a $26.4 \%$ yield. LC-MS $\left(\mathrm{C}_{26} \mathrm{H}_{35} \mathrm{~N}_{5} \mathrm{O}_{5}\right)$ : $[\mathrm{M}+\mathrm{H}]^{+}=498.2467$ (m/z), calc: 497.2638 .

\section{Preparation of NEB}

To a 20 -mL glass vial containing $2.5 \mathrm{mg}$ of NOTA-tolidine $(5.0 \mu \mathrm{mol})$ in $0.3 \mathrm{~mL}$ of water was added $18 \mu \mathrm{mol}$ of $\mathrm{HCl}$ in $0.1 \mathrm{~mL}$ of water. The mixture was cooled in an ice bath, and $0.5 \mathrm{mg}$ of sodium nitrite $(7.2 \mu \mathrm{mol})$ in $0.1 \mathrm{~mL}$ of water was added to the vial. The mixture was stirred in an ice bath for $20 \mathrm{~min}$, and the yellow diazonium salt solution was added dropwise to another vial in an ice bath containing $4.0 \mathrm{mg}$ of 1-amino8-naphthol-2,4-disulfonic acid $(10.0 \mu \mathrm{mol})$ and $2.4 \mathrm{mg}$ of sodium bicarbonate $(28.5 \mu \mathrm{mol})$ in $0.2 \mathrm{~mL}$ of water. The mixture was stirred in an ice bath for $2 \mathrm{~h}$ and purified with semipreparative HPLC. The product (denoted as NEB) was collected $\left(\mathrm{R}_{\mathrm{t}}=19.0 \mathrm{~min}\right.$ ) and lyophilized overnight to give $1.4 \mathrm{mg}$ of pure product in a $46.6 \%$ yield. LC-MS $\left(\mathrm{C}_{36} \mathrm{H}_{41} \mathrm{~N}_{7} \mathrm{O}_{12} \mathrm{~S}_{2}\right)$ : $[\mathrm{M}-\mathrm{H}]^{-}=826.2415(\mathrm{~m} / \mathrm{z})$, calc: 827.2255 .

\section{Preparation of ${ }^{18} \mathrm{~F}$-AIF-NEB}

To a 1-mL plastic tube containing $3 \mu \mathrm{L}$ of $2 \mathrm{mM}$ aluminum chloride in $0.5 \mathrm{M}, \mathrm{pH} 4.0$, sodium acetate buffer and $6 \mu \mathrm{L}$ of $3 \mathrm{mM} \mathrm{NEB}$ in $0.5 \mathrm{M}, \mathrm{pH} 4.0$, sodium acetate buffer was added $0.13 \mathrm{~mL}$ of acetonitrile and $0.05 \mathrm{~mL}$ of aqueous ${ }^{18} \mathrm{~F}$-fluoride $(0.3-0.9 \mathrm{GBq})$. The mixture was stirred in a vortex mixer and heated in a $105^{\circ} \mathrm{C}$ heating block for $10 \mathrm{~min}$. The vial was cooled, and the solution was diluted with $10 \mathrm{~mL}$ of water and trapped on a Varian Bond Elut $\mathrm{C}_{18}$ column $(100 \mathrm{mg})$. The radioactivity trapped on the $\mathrm{C}_{18}$ column was eluted with $0.3 \mathrm{~mL}$ of $80 \%$ ethanol/water containing $1 \mathrm{mM} \mathrm{HCl}$. The ethanol solution was evaporated with argon flow, and the final product was dissolved in phosphate-buffered saline and analyzed by HPLC.

\section{Preparation of ${ }^{18} \mathrm{~F}$-Fluorobenzyl (FB)-Albumin}

${ }^{18} \mathrm{~F}$-SFB was prepared with an Eckert \& Ziegler synthesizer according to a published procedure (22). The HPLC-purified ${ }^{18} \mathrm{~F}-\mathrm{SFB}$ was trapped on a Waters $\mathrm{C}-18$ cartridge and eluted with $1 \mathrm{~mL}$ of methylene chloride into a $1-\mathrm{mL}$ plastic tube. For a typical run, after evaporation of the solvent, the radioactivity (148 MBq) was redissolved in $5 \mu \mathrm{L}$ of acetonitrile, and $0.5 \mathrm{mg}$ of mouse serum albumin (MSA) in $100 \mu \mathrm{L}$ of $\mathrm{pH} 8.5$ borate buffer was added to the tube and reacted at $37^{\circ} \mathrm{C}$ for $10 \mathrm{~min}$. The reaction mixture was purified on a PD-10 size-exclusion column (GE Healthcare) to give $92.5 \mathrm{MBq}$ of product in a $62.5 \%$ radiochemical (non-decay-corrected) yield.

\section{Preparation of ${ }^{64} \mathrm{Cu}-\mathrm{NEB}$}

To a 1-mL plastic tube containing $11.0 \mu \mathrm{g}$ of NEB in $100 \mu \mathrm{L}$ of 0.4 $\mathrm{M}, \mathrm{pH} 5.5$, sodium acetate buffer was added $5 \mu \mathrm{L}$ of aqueous ${ }^{64} \mathrm{Cu}-$ $\mathrm{CuCl}_{2}$ solution $(262.7 \mathrm{MBq})$. The mixture was stirred in a vortex mixer and heated on an $80^{\circ} \mathrm{C}$ heating block for $10 \mathrm{~min}$. The tube was cooled and the radioactive solution transferred to a $10-\mathrm{mL}$ syringe containing $10 \mathrm{~mL}$ of water. This solution was passed through a Varian Bond Elut $\mathrm{C}_{18}$ cartridge $(100 \mathrm{mg})$, and the desired product was trapped on the cartridge. The radioactivity trapped on the $\mathrm{C}_{18}$ column was eluted with $0.45 \mathrm{~mL}$ of $80 \%$ ethanol/water with $1 \mathrm{mM} \mathrm{HCl}$ to give $185 \mathrm{MBq}$ of the desired product in a $70 \%$ radiochemical yield. The ethanol solution was evaporated with argon flow, and the final product was dissolved in phosphate-buffered saline and analyzed by HPLC. The radiochemical purity was greater than $95 \%$.

\section{Preparation of Animal Models}

All animal studies were conducted in accordance with the principles and procedures outlined in the Guide for the Care and Use of Laboratory Animals (23) and were approved by the Institutional Animal Care and Use Committee of the Clinical Center, National Institutes of Health. The UM-22B human head and neck carcinoma cancer cell line was grown in Dulbecco modified Eagle medium supplemented with $10 \%$ fetal bovine serum, penicillin $(100 \mathrm{IU} / \mathrm{mL})$, and streptomycin $(100 \mu \mathrm{g} / \mathrm{mL})$ (Invitrogen) and in a humidified atmosphere containing $5 \% \mathrm{CO}_{2}$ at $37^{\circ} \mathrm{C}$. The tumor model was developed in 5- to 6-wk-old female athymic nude mice (Harlan Laboratories) by injection of $5 \times 10^{6}$ cells into their right shoulders. The mice underwent small-animal PET studies when the tumor volume reached $100-300 \mathrm{~mm}^{3}$ (2-3 wk after inoculation).

The myocardial infarction (MI) model was prepared in male BALB/c mice aged $8-10 \mathrm{wk}$. MI was induced by ligation of the left anterior descending coronary artery $1-3 \mathrm{~mm}$ from the tip of the left auricle with a 7-0 polypropylene suture. The occlusion and reperfusion were confirmed by ST-segment elevation on an electrocardiogram monitor (EC-60 model; Silogic) after surgery. For mice in the control group, the surgery was performed but without ligation of the left coronary artery.

In Vivo Labeling of Albumin • Niu et al. 
The acute inflammation model was prepared by intramuscular injection of turpentine. Up to $30 \mu \mathrm{L}$ of turpentine were injected in the caudal thigh muscles of the left hind limb. The PET imaging was performed $24 \mathrm{~h}$ after turpentine injection.

\section{Small-Animal PET Imaging and Analysis}

PET scans were obtained and image analysis performed using an Inveon small-animal PET scanner (Siemens Medical Solutions). About 3.7 MBq of ${ }^{18} \mathrm{~F}$-AlF-NEB or ${ }^{18} \mathrm{~F}-\mathrm{FB}$-albumin or $7.4 \mathrm{MBq}$ of ${ }^{64} \mathrm{Cu}-\mathrm{NEB}$ were administered via tail vein injection under isoflurane anesthesia. For normal mice, 60-min dynamic PET scans were acquired. For tumor mice, 60-min dynamic PET scans were acquired, followed by a series of late-time-point scans at 2,4 , and $24 \mathrm{~h}\left({ }^{64} \mathrm{Cu}\right.$ only) after tracer injection. With the acute inflammation model, 5-min static PET images were acquired at $30 \mathrm{~min}, 1 \mathrm{~h}$, and $2 \mathrm{~h}$ after injection $(n=3-5$ per group). The images were reconstructed using a 2-dimensional orderedsubset expectation maximum algorithm, and no correction was applied for attenuation or scatter. For each scan, regions of interest (ROIs) were drawn using vendor software (ASI Pro 5.2.4.0; Siemens Medical Solutions) on decay-corrected whole-body coronal images. The radioactivity concentrations (accumulation) within the heart, muscle, liver, and kidneys were obtained from mean pixel values within the multiple ROI volumes and then converted to megabecquerel per milliliter. These values were then divided by the administered activity to obtain (assuming a tissue density of $1 \mathrm{~g} / \mathrm{mL}$ ) an image-ROI-derived percentage injected dose per gram (\%ID/g).

For electrocardiography (ECG)-gated PET studies, mice were imaged prone within the PET scanner and kept at $37^{\circ} \mathrm{C}$ using a heating pad with continuous rectal measurement of body temperature. ECG electrodes were placed on the forepaws and the left hind paw. Respiration was measured using a small pressure detector lying under the thorax of the mice. The cardiac excitation and respiration were recorded with a Biovet system (Spin Systems Pty Ltd.) throughout the scan. A list-mode PET scan of 30 min was acquired at 15 min after intravenous injection of around $3.7 \mathrm{MBq}$ of ${ }^{18} \mathrm{~F}-\mathrm{NEB}$ (both control and MI models), respectively. The cardiac cycle from the ${ }^{18} \mathrm{~F}-\mathrm{NEB}$ list-mode acquisitions was separated into 8 equal intervals using the Siemens Inveon Acquisition Workplace and reconstructed using 3-dimensional ordered-subset expectation maximum with 4 iterations and 3-dimensional maximum a posteriori (MAP) reconstruction with 32 iterations. The measurements of left-ventricular function from the PET datasets were calculated and quantified using the Inveon Research Workplace (Siemens Preclinical Solutions). A 55\% intensity threshold was used for the quantitation of left ventricular ejection fraction.

\section{Hematoxylin and Eosin Staining}

Tissues were collected and fixed in Z-fix (buffered zinc formalin fixatives; Anatech) for at least $24 \mathrm{~h}$ and then embedded in paraffin for sectioning. The slices $(10 \mu \mathrm{m})$ were stained with hematoxylin and eosin by standard techniques. The stained tissue sections were observed with a BX41 bright field microscopy (Olympus).

\section{Statistical Analysis}

Quantitative data were expressed as mean \pm SD. Means were compared using the Student $t$ test provided by Excel (Microsoft) or GraphPad Prism (GraphPad Software, Inc.). A $P$ value of less than 0.05 was considered statistically significant.

\section{RESULTS}

\section{Chemistry and Radiochemistry}

To facilitate radioactive fluorine labeling of the albuminbinding compound, we synthesized NEB in 2 steps starting from o-tolidine as shown in Figure 1. The NOTA was first coupled to o-tolidine using diethyl cyanophosphonate to give NOTA-o-tolidine in a $26 \%$ yield after preparative reversed-phase HPLC. NOTA-otolidine was then coupled to 1-amino-8-naphthol-2,4-disulfonic acid to give NEB through the formation of diazonium salt with a yield of $46.6 \%$. The purity of the product was greater than $98 \%$ based on HPLC analysis, and the identity of the product was confirmed by LC-MS. This truncated version of EB retained the binding ability with albumin because stable NEB-albumin complex was confirmed by LC-MS (Supplemental Fig. 1; supplemental materials are available at http://jnm.snmjournals.org). The complex formation was also confirmed by saturation binding assay. The bound NEB could be separated from the unbound NEB using agarose gel electrophoresis. Quantitation of the mass of unbound NEB as a function of its concentration allowed determination of the dissociation constant $(48.9 \pm 3.81 \mu \mathrm{M})$ (Supplemental Fig. 2).

The procedure for radiolabeling of NEB is shown in Figure 1. The radiochemical yield for ${ }^{18} \mathrm{~F}-\mathrm{AlF}-\mathrm{NEB}$ was $58.4 \% \pm$ $11.3 \%(n=5)$, with a total synthesis and work-up time of 20-30 min. A single peak was detected on thin-layer chromatography, and the radiochemical purity was greater than $95 \%$ based on HPLC analysis (Supplemental Figs. 3 and 4). Labeling of NEB with ${ }^{64} \mathrm{Cu}$ also took $20-30 \mathrm{~min}$, with a radiochemical yield of $74 \%$. For in vitro labeling of albumin, ${ }^{18} \mathrm{~F}-\mathrm{SFB}$ was first prepared and purified by HPLC and then used for protein labeling. The whole labeling process took about 2-3 h, with a radiochemical purity of greater than $95 \%$. Both ${ }^{18} \mathrm{~F}-\mathrm{AlF}-\mathrm{NEB}$ and ${ }^{64} \mathrm{Cu}-\mathrm{NEB}$ showed good stability in mouse serum even after $120 \mathrm{~min}$ incubation at $37^{\circ} \mathrm{C}$ (Supplemental Figs. 5 and 6).

FIGURE 1. Synthesis and ${ }^{18} \mathrm{~F}$-aluminum fluoride radiolabeling of NEB. 


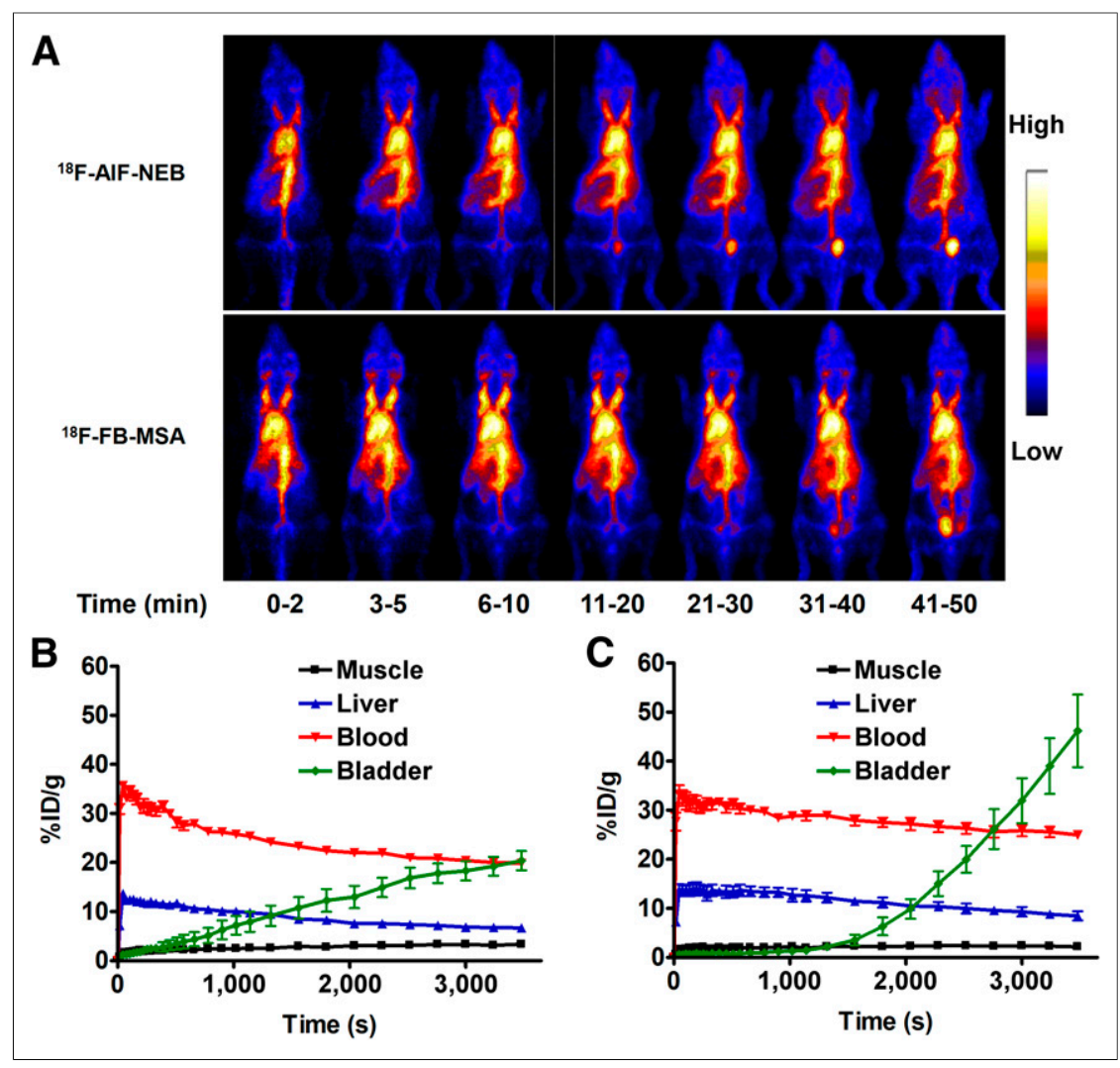

FIGURE 2. (A) Series of maximum-intensity-projection PET images in normal mice after intravenous injection of either ${ }^{18} \mathrm{~F}-\mathrm{AlF}-\mathrm{NEB}$ or ${ }^{18} \mathrm{~F}-\mathrm{FB}-\mathrm{MSA}$. Each mouse received around $3.7 \mathrm{MBq}$ of radioactivity. Images were reconstructed from a 60-min dynamic scan. (B) Time-activity curves of ROls outlined over muscle, heart, liver, and bladder regions on ${ }^{18} \mathrm{~F}$-AIF-NEB PET images. (C) Time-activity curves of ROls outlined over muscle, heart, liver, and bladder regions on ${ }^{18} \mathrm{~F}-\mathrm{FB}-\mathrm{MSA}$ PET images.

in the bladder increased continuously with time (Fig. 2A). HPLC analysis of urine samples found that the radioactivity came from both intact ${ }^{18} \mathrm{~F}$-AlF-NEB and its metabolites. In blood samples, most of the radioactivity was from the intact ${ }^{18} \mathrm{~F}$-AlF-NEB (Supplemental Fig. 7).

We also compared in vivo labeling by ${ }^{18} \mathrm{~F}-$ AlF-NEB with in vitro-labeled ${ }^{18} \mathrm{~F}-\mathrm{FB}-\mathrm{MSA}$. The PET results showed similar distribution patterns between the 2 probes. The blood and liver time-activity curves from ${ }^{18} \mathrm{~F}-\mathrm{FB}-\mathrm{MSA}$ showed slightly less but nonsignificant $(P>0.05)$ decline rates than those of ${ }^{18} \mathrm{~F}$ AlF-NEB. The bladder showed almost no uptake of ${ }^{18}$ F-FB-MSA within the first $20 \mathrm{~min}$ but increased dramatically afterward. At 60 min after injection of ${ }^{18} \mathrm{~F}-\mathrm{FB}-\mathrm{MSA}$, the radioactivity in the bladder was much higher than that in the blood (Figs. 2B and 2C).

After PET imaging, the animals were sacrificed and major tissues and organs were collected. The radioactivity was measured, and the results are presented in Table 1. With both ${ }^{18} \mathrm{~F}-\mathrm{AlF}-\mathrm{NEB}$ and ${ }^{18} \mathrm{~F}-\mathrm{FB}-\mathrm{MSA}$, the blood had the highest counts. The radioactivity levels in the liver, kidneys, and spleen for ${ }^{18} \mathrm{~F}$-FB-MSA (in vitro labeling) were significantly higher than those for ${ }^{18} \mathrm{~F}$-AlF-NEB (in vivo labeling).

\section{ECG-Gated PET Imaging}

One of the major applications of bloodpool imaging is to evaluate cardiac function.

\section{In Vivo PET Imaging of Normal Mice}

The in vivo pharmacokinetics of ${ }^{18} \mathrm{~F}-\mathrm{AlF}-\mathrm{NEB}$ were evaluated with dynamic PET in healthy BALB/C mice. As expected, most of the radioactivity was retained in the circulation system during the first $60 \mathrm{~min}$ after injection. Ventricles of the heart and major arteries were clearly visualized on PET images. The locations of liver, kidneys, and spleen were also identified because of the abundant blood supply of these organs (Fig. 2A). On the basis of the timeactivity curves generated by PET images, whole-blood radioactivity was decreased only by $10 \%$ from 10 to $60 \mathrm{~min}$ after injection. Organs with a large blood volume such as liver, spleen, and kidneys showed higher uptake than the skeletal muscle but were considerably less than the blood cavities within the heart and main branches of the blood vessels. Counts in the heart volume of interest peaked rapidly at $1 \mathrm{~min}$ after injection and then declined gradually but remained higher than those in the liver and kidneys. The radioactivity
We first tested the probe in normal Sprague-Dawley rats (Supplemental Fig. 9). On the representative transaxial and coronal images of ${ }^{18} \mathrm{~F}-\mathrm{AlF}-\mathrm{NEB}$, the fine septal borders between the left and right ventricles were clearly identified. The major arteries and vena cava were also visualized. After dividing each cardiac cycle into 8 fragments, the end-systole and end-diastole can be easily distinguished.

We also developed a mouse MI model and performed ECGgated PET with the similar procedure. The cardiac cycle with 8 fragmentations is presented in Figure 3A. Although limited by the much smaller size of the mouse heart and spatial resolution of PET, the ventricles can be clearly distinguished. The end-systole volume of MI mice was significantly higher than that in the control mice. On the basis of PET images, left ventricular volume over the cardiac cycle was generated and is shown in Figure 3B. The MI mice showed much lower left ventricular ejection fraction than the control

TABLE 1

Biodistribution of ${ }^{18} \mathrm{~F}-\mathrm{AIF}-\mathrm{NEB}$ and ${ }^{18} \mathrm{~F}-\mathrm{FB}-\mathrm{MSA}$ in Normal BALB/c Mice at 60 Minutes After Tracer Injection

\begin{tabular}{cccccccc}
\hline Tracer & Blood & Muscle & Liver & Kidney & \multicolumn{1}{c}{ Spleen } & Pancreas & Intestine \\
\hline${ }^{18}$ F-AlF-NEB & $26.35 \pm 1.52$ & $1.80 \pm 0.48$ & $5.05 \pm 0.13$ & $6.80 \pm 1.16$ & $3.75 \pm 0.48$ & $2.17 \pm 1.38$ & $4.42 \pm 3.00$ \\
${ }^{18}$ F-FB-MSA & $34.71 \pm 3.29$ & $0.80 \pm 0.09$ & $7.42 \pm 0.53$ & $12.01 \pm 5.59$ & $8.52 \pm 1.71$ & $2.09 \pm 0.15$ & $3.10 \pm 0.28$
\end{tabular}

Data are mean $\pm S D, \% I D / g$. 


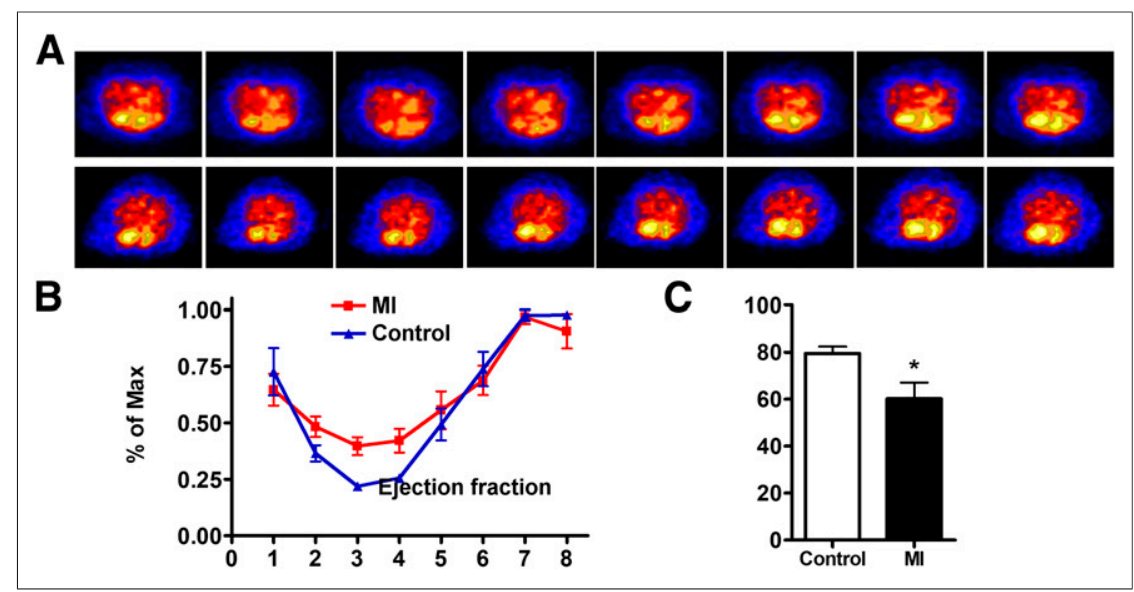

FIGURE 3. (A) ECG-gated blood-pool imaging of control and MI mice. Transaxial images were reconstructed to display 8 intervals of 1 cardiac cycle. (B) Left ventricular volume curve calculated from PET. (C) Left ventricular ejection fraction calculated from ECG-gated PET. Max = maximum. ${ }^{\star} P<0.05$

mice $(79.54 \% \pm 2.95 \%$ vs. $60.24 \% \pm 6.88 \%, P<0.01)$ (Fig. $3 \mathrm{C})$. We also performed ultrasound with the same 2 groups of mice. The left ventricular ejection fraction results determined by ultrasound were consistent with those from PET imaging (Supplemental Fig. 10).

\section{PET Imaging of Vascular Leakage}

We also developed an acute skeletomuscular inflammation model by local intramuscular injection of turpentine and performed ${ }^{18} \mathrm{~F}$ AlF-NEB PET to evaluate the images for vascular leakage. The inflammation induced by local intramuscular injection of turpentine was indicated by neutrophil infiltration on hematoxylin and eosin staining (Fig. 4A). As shown in Figure 4B, high level of radioactivity accumulation was observed within inflamed muscles at 1 and $2.5 \mathrm{~h}$ after ${ }^{18} \mathrm{~F}-\mathrm{AlF}-\mathrm{NEB}$ administration. Indicated by the timeactivity curves of 60-min dynamic imaging, the tracer uptake in the inflammatory muscles increased gradually along with time whereas no apparent changes were observed in time-activity curves of the collateral muscles (Fig. 4C). Quantitative analysis of PET images indicated an uptake of $5.94 \pm 0.69 \% \mathrm{ID} / \mathrm{g}$ at $1 \mathrm{~h}$ after ${ }^{18} \mathrm{~F}-\mathrm{AlF}-\mathrm{NEB}$ injection and $7.50 \pm 0.69 \% \mathrm{ID} / \mathrm{g}$ at $2.5 \mathrm{~h}$ after injection (Fig. 4D).

\section{PET Imaging of Tumor Vasculature}

Malignant tumors are characterized by torturous blood vessels and high vascular permeability (24). Besides, the antiangiogenesis and normalization of tumor vasculature have been intensively investigated (25). Unlike radiolabeled $\mathrm{RBCs}$, albumin can be used to study vascular permeability in tumors. We labeled NEB with ${ }^{64} \mathrm{Cu}$, a positron emitter with longer half-life than ${ }^{18} \mathrm{~F}(12.6 \mathrm{~h}$ vs. $109.8 \mathrm{~min}$, respectively). We developed a UM-22B xenograft model and obtained 60-min dynamic scans and then static scans at late time points up to $24 \mathrm{~h}$. The in vivo distribution of ${ }^{64} \mathrm{Cu}-$ NEB was similar to that of ${ }^{18} \mathrm{~F}-\mathrm{AlF}-\mathrm{NEB}$, with most of the radioactivity retained in the circulation system (Fig. 5A). Even at $4 \mathrm{~h}$ after tracer injection, radioactivity within the heart region and major vessels was still dominant. The tumor uptake was $5.73 \pm$ $1.11 \% \mathrm{ID} / \mathrm{g}$ at $1 \mathrm{~h}$ after injection and increased to $8.03 \pm 0.77$ $\% \mathrm{ID} / \mathrm{g}$ at $2 \mathrm{~h}$ after injection. At $24 \mathrm{~h}$ after tracer injection, the tumor uptake was still at a relatively high level $(8.07 \pm 1.01 \% \mathrm{ID} / \mathrm{g})$. The tracer uptake over the heart region was $16.09 \pm 0.51 \% \mathrm{ID} / \mathrm{g}$ at $1 \mathrm{~h}$ after injection, dropping to $8.58 \pm 0.81 \% \mathrm{ID} / \mathrm{g}$ at $24 \mathrm{~h}$ (Fig. $5 \mathrm{~B}$ ).

\section{DISCUSSION}

To meet the requirement for both clinical application and preclinical research, herein, we investigated a fast in vivo albuminlabeling method for PET imaging. The preparation procedure is rapid and efficient. PET images using in vivo-labeled albumin through a newly developed truncated EB derivative, ${ }^{18} \mathrm{~F}-\mathrm{AlF}-\mathrm{NEB}$, are comparable to those using in vitro-labeled albumin through ${ }^{18} \mathrm{~F}-\mathrm{FB}-$ albumin. The in vivo-labeling strategy can be applied to blood-pool imaging to evaluate cardiac function under both physiologic and pathologic conditions. This method can also be used to evaluate vascular permeability in tumors, inflammatory diseases, and ischemic or infarcted lesions. Because of the simple synthesis procedure, this novel PET tracer has great potential for clinical translation. ${ }^{18} \mathrm{~F}$-AlF-NEB would be an ideal alternative to radiolabeled RBCs for blood-pool imaging because autologous blood products present significant risks to both the operator handling the product and the patient receiving it.

As expected, most of the radioactivity was retained in the circulatory system after intravenous injection of ${ }^{18} \mathrm{~F}-\mathrm{AlF}-\mathrm{NEB}$, justifying the feasibility of using this tracer as a blood-pool imaging agent. Immediately after intravenous injection of ${ }^{18} \mathrm{~F}-\mathrm{AlF}$ NEB, a small amount of tracer was rapidly cleared out of circulation before binding to albumin. We also cannot exclude the possibility of dissociation of the NEB-albumin complex, especially at the early phase, because the dissociation constant of NEB to albumin is around $50 \mu \mathrm{M}$. However, because of the low amount of NEB injected and

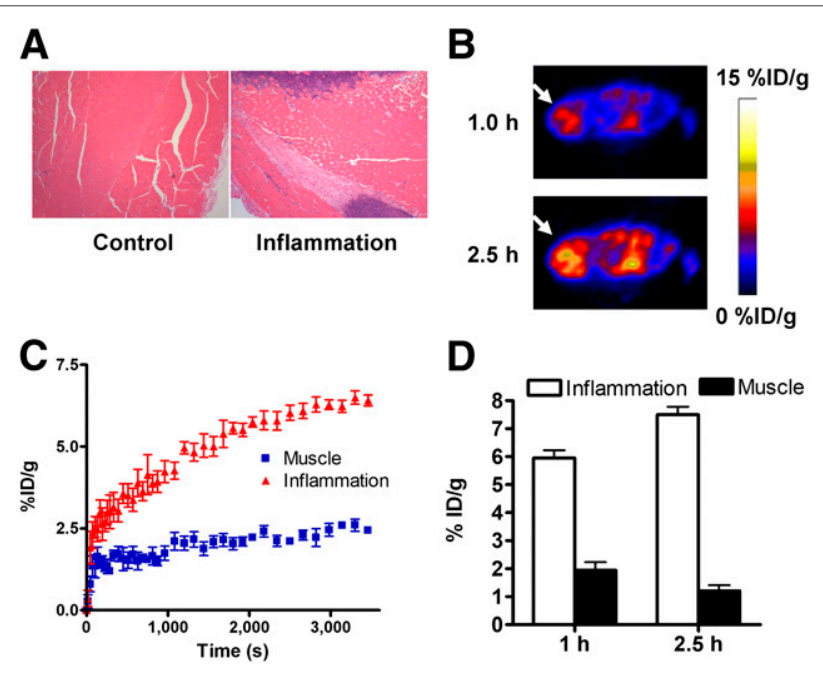

FIGURE 4. (A) Hematoxylin and eosin staining of inflammatory muscles at $24 \mathrm{~h}$ after local injection of turpentine oil. (B) Transaxial PET images of mice that received turpentine oil injection. Ten-min static PET scans were obtained after intravenous injection of $3.7 \mathrm{MBq}$ of ${ }^{18} \mathrm{~F}-\mathrm{AlF}-\mathrm{NEB}$. Apparent radioactivity accumulation was observed in inflamed muscles at both 1 and $2.5 \mathrm{~h}$ after tracer injection, as indicated by arrows. (C) Time-activity curves over inflamed and contralateral healthy muscles based on PET images from a 60-min dynamic scan using ${ }^{18} \mathrm{~F}-\mathrm{AlF}-\mathrm{NEB}$. (D) Quantitative analysis of ${ }^{18} \mathrm{~F}$-AIF-NEB uptake in both inflamed and contralateral healthy muscles at 1 and $2.5 \mathrm{~h}$ after tracer injection. 


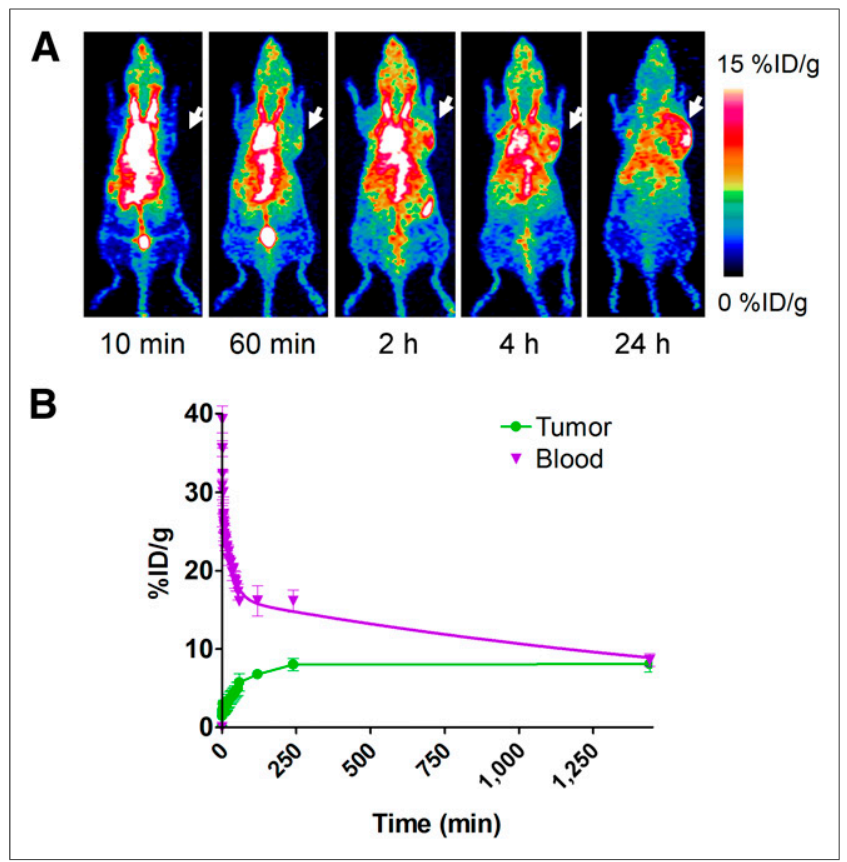

FIGURE 5. (A) Series of maximum-intensity-projection PET images of UM-22B tumor-bearing mice after intravenous injection of ${ }^{64} \mathrm{Cu}$-NEB. Tumors were indicated by arrows. (B) Time-activity curves of ROls over heart and tumor regions.

highly abundant reservoir of albumin protein, we are not concerned about the complex formation and its stability in the blood circulation. Indeed, from 15 to $60 \mathrm{~min}$ after injection, the tracer showed a much slower clearance of radioactivity from the blood. We believe that this down slope was caused mainly by the turnover of albumin from blood circulation to the interstitial space. The in vitro-labeled albumin ( $\left.{ }^{18} \mathrm{~F}-\mathrm{FB}-\mathrm{MSA}\right)$ also showed a similar clearance pattern within the same time range. In fact, the tendency of declining uptake of albumin-based tracer concentration in the blood pool has also been observed in other studies, in which the albumin was labeled in vitro $(4,26)$. Directly labeled albumin showed significantly higher liver and spleen uptake than in vivo ${ }^{18} \mathrm{~F}-\mathrm{AlF}-\mathrm{NEB}$ (Table 1). It is highly possible that the structure of albumin was compromised by the in vitro-labeling process (11).

As the most widely used PET imaging probe approved by the Food and Drug Administration, ${ }^{18}$ F-FDG has been used for ECGgated PET imaging to evaluate cardiac function (27). The parameters from ${ }^{18} \mathrm{~F}$-FDG PET are comparable with those from MR imaging and CT studies. However, blood-pool imaging probes such as ${ }^{68}$ Ga-DOTA-albumin have shown advantages over ${ }^{18}$ F-FDG PET because in the infarcted heart, the cardiac wall is not intact due to decreased or null uptake of ${ }^{18} \mathrm{~F}-\mathrm{FDG}$, leading to imprecise delineation of the infarcted myocardium (28). In this study, we successfully visualized the cardiac ventricles and major vessels using ${ }^{18} \mathrm{~F}-\mathrm{AlF}-$ NEB PET. After each cardiac cycle was divided into 8 equal time intervals, the volume of left ventricle in each interval can be quantified to calculate the ejection fraction. Because of the limitation of spatial resolution and partial-volume effect, the left ventricular ejection fraction in MI mice based on PET quantification is higher than that from ultrasound. Besides, the accuracy of ECG-gated PET imaging is also affected by the reconstruction algorithm (29), the software used for outlining ROIs, and ECG gating (26). Therefore, further investigations are needed to optimize the conditions of ECG-gated ${ }^{18} \mathrm{~F}-\mathrm{AlF}-\mathrm{NEB}$ PET for both preclinical studies and clinical applications.

Other than being a blood-pool imaging agent, fluorescence dye or radionuclide-labeled albumin conjugates have also been extensively used to image increased vascular permeability in various inflammatory diseases (30). In this study, we applied ${ }^{18} \mathrm{~F}$-AlF-NEB to evaluate increased vascular permeability in both turpentine-induced acute inflammation and xenografted tumor models. The inflamed muscle showed continuously increased radioactivity accumulation, indicating the leakage of serum albumin into surrounding interstitial tissues. The late-time-point scans also provided better contrast between inflammatory muscles and contralateral normal muscle, which is consistent with the results reported previously with in vitrolabeled albumin (30).

Malignant tumors often show increased uptake and retention of high-molecular-weight nontargeted drugs and prodrugs, which is known as the enhanced permeability and retention effect (31). Moreover, angiogenesis and vasculature vary in different tumor types (32). Thus, the ability to noninvasively evaluate tumor vasculature and permeability would be helpful for patient preselection and therapy response monitoring. With ${ }^{64} \mathrm{Cu}-\mathrm{NEB}$ PET, we found that UM-22B tumors are permeable, which is in accordance with our previous report (15). We noticed that the time-activity curve over the heart region showed a down slope, and the curve over tumor region showed a plateau. The difference between these 2 slopes was thus used to quantify tumor vascular permeability.

EB has been used clinically to evaluate the blood volume (33). The clinical practice has been discontinued because of the toxicity of vital dye, especially potential pulmonary embolism after intravenous injection. The mechanism of this toxicity is due to an EB dose-related induction of platelet aggregation, which begins with the threshold concentration of about $100 \mu \mathrm{M}$ (34). For in vivo PET imaging, only a trace amount of ${ }^{18}$ F-AlF-NEB $(\sim 3 \mu \mathrm{g} / \mathrm{mL}$ or 3.6 $\mu \mathrm{M}$ ) was used, which would not elicit toxicity. Even with multiple injections, the dose will still be safe. Of course, a more robust toxicity study is still needed before clinical translation of ${ }^{18} \mathrm{~F}-\mathrm{AlF}$ NEB. We also performed a dosimetry study based on the PET imaging data. For the sensitive organs such as red bone marrow, the absorbed dose is $0.23 \mathrm{mSv}$ for ${ }^{18} \mathrm{~F}-\mathrm{AlF}-\mathrm{NEB}$ and $6.14 \mathrm{mSv}$ for ${ }^{64} \mathrm{Cu}-\mathrm{NEB}$ if we inject $185 \mathrm{MBq}$ of radioactivity to each subject (Supplemental Tables 1 and 2). This dose allows multiple PET scans without exceeding the recommended annual dose limit for diagnostic purposes. Future large-animal and human scans will provide a more accurate estimate of the dosimetry.

\section{CONCLUSION}

In this study, for the first time to our knowledge, we reported an in vivo albumin-labeling method with a NOTA-conjugated truncated EB dye derivative. The tracers could be used to visualize the distribution and local accumulation of serum albumin noninvasively by PET. ECG-gated ${ }^{18}$ F-AlF-NEB PET could be used to evaluate the loss of cardiac function in mice with MI. The vascular leakage induced by acute inflammation and increased permeability in malignant tumors could also be visualized and quantified with this strategy. The simple labeling strategy and broad applications of these imaging probes will likely make them useful for clinical use.

\section{DISCLOSURE}

The costs of publication of this article were defrayed in part by the payment of page charges. Therefore, and solely to indicate this 
fact, this article is hereby marked "advertisement" in accordance with 18 USC section 1734. This work was supported by the Intramural Research Program of the National Institute of Biomedical Imaging and Bioengineering (NIBIB), National Institutes of Health (NIH). No other potential conflict of interest relevant to this article was reported.

\section{REFERENCES}

1. Elsadek B, Kratz F. Impact of albumin on drug delivery: new applications on the horizon. J Control Release. 2012;157:4-28.

2. Klohs J, Steinbrink J, Bourayou R, et al. Near-infrared fluorescence imaging with fluorescently labeled albumin: a novel method for non-invasive optical imaging of blood-brain barrier impairment after focal cerebral ischemia in mice. J Neurosci Methods. 2009;180:126-132.

3. McAfee JG, Stern HS, Fueger GF, Baggish MS, Holzman GB, Zolle I. ${ }^{99 m}$-Tc labeled serum albumin for scintillation scanning of the placenta. J Nucl Med. 1964;5:936-946.

4. Hoffend J, Mier W, Schuhmacher J, et al. Gallium-68-DOTA-albumin as a PET blood-pool marker: experimental evaluation in vivo. Nucl Med Biol. 2005;32:287-292.

5. Lauffer RB, Parmelee DJ, Dunham SU, et al. MS-325: albumin-targeted contrast agent for MR angiography. Radiology. 1998;207:529-538.

6. Kearfott KJ. Absorbed dose estimates for positron emission tomography (PET): $\mathrm{C}^{15} \mathrm{O},{ }^{11} \mathrm{CO}$, and $\mathrm{CO}^{15} \mathrm{O}$. J Nucl Med. 1982;23:1031-1037.

7. Herance JR, Gispert JD, Abad S, et al. Erythrocytes labeled with $\left[{ }^{18} \mathrm{~F}\right] \mathrm{SFB}$ as an alternative to radioactive $\mathrm{CO}$ for quantification of blood volume with PET. Contrast Media Mol Imaging. 2013;8:375-381.

8. Okazawa H, Yonekura Y, Fujibayashi Y, et al. Measurement of regional cerebral plasma pool and hematocrit with copper-62-labeled HSA-DTS. J Nucl Med. 1996;37:1080-1085.

9. Anderson CJ, Rocque PA, Weinheimer CJ, Welch MJ. Evaluation of copperlabeled bifunctional chelate-albumin conjugates for blood pool imaging. $\mathrm{Nucl}$ Med Biol. 1993;20:461-467.

10. Kilbourn MR, Dence CS, Welch MJ, Mathias CJ. Fluorine-18 labeling of proteins. J Nucl Med. 1987;28:462-470.

11. Wängler B, Quandt G, Iovkova L, et al. Kit-like ${ }^{18} \mathrm{~F}$-labeling of proteins: synthesis of 4-(di-tert-butyl $\left[{ }^{18} \mathrm{~F}\right]$ fluorosilyl)benzenethiol (Si $\left.\left[{ }^{18} \mathrm{~F}\right] \mathrm{FA}-\mathrm{SH}\right)$ labeled rat serum albumin for blood pool imaging with PET. Bioconjug Chem. 2009;20: 317-321.

12. He XM, Carter DC. Atomic structure and chemistry of human serum albumin. Nature. 1992;358:209-215.

13. Spahr PF, Edsall JT. Amino acid composition of human and bovine serum mercaptalbumins. J Biol Chem. 1964;239:850-854.

14. Phillips RA, Yeomans A, Dole VP, Farr LE, Van Slyke DD, Hogan D. Estimation of blood volume from change in blood specific gravity following a plasma infusion. J Clin Invest. 1946;25:261-269.

15. Niu G, Li Z, Xie J, Le QT, Chen X. PET of EGFR antibody distribution in head and neck squamous cell carcinoma models. J Nucl Med. 2009;50:1116-1123.
16. Yan J, Manaenko A, Chen S, et al. Role of SCH79797 in maintaining vascular integrity in rat model of subarachnoid hemorrhage. Stroke. 2013;44:1410-1417.

17. Patterson CE, Rhoades RA, Garcia JG. Evans blue dye as a marker of albumin clearance in cultured endothelial monolayer and isolated lung. J Appl Physiol. 1992;72:865-873.

18. Moitra J, Sammani S, Garcia JG. Re-evaluation of Evans blue dye as a marker of albumin clearance in murine models of acute lung injury. Transl Res. 2007;150: 253-265.

19. Lang L, Li W, Guo N, et al. Comparison study of $\left[{ }^{18} \mathrm{~F}\right] \mathrm{FAl}-\mathrm{NOTA}-\mathrm{PRGD} 2,\left[{ }^{18} \mathrm{~F}\right]$ FPPRGD2, and $\left[{ }^{68} \mathrm{Ga}\right] \mathrm{Ga}-N O T A-P R G D 2$ for PET imaging of U87MG tumors in mice. Bioconjug Chem. 2011;22:2415-2422.

20. McBride WJ, D'Souza CA, Sharkey RM, et al. Improved F-18 labeling of peptides with a fluoride-aluminum-chelate complex. Bioconjug Chem. 2010; 21:1331-1340.

21. McBride WJ, Sharkey RM, Karacay H, et al. A novel method of F-18 radiolabeling for PET. J Nucl Med. 2009;50:991-998.

22. Chen X, Park R, Hou Y, et al. MicroPET imaging of brain tumor angiogenesis with ${ }^{18}$ F-labeled PEGylated RGD peptide. Eur J Nucl Med Mol Imaging. 2004;31:1081-1089.

23. Guide for the Care and Use of Laboratory Animals. Bethesda, MD: National Institutes of Health; 1985. NIH publication 85-23.

24. Folkman J. Tumor angiogenesis: therapeutic implications. $N$ Engl J Med. 1971;285:1182-1186.

25. Bergers G, Javaherian K, Lo KM, Folkman J, Hanahan D. Effects of angiogenesis inhibitors on multistage carcinogenesis in mice. Science. 1999;284:808-812.

26. Todica A, Brunner S, Boning G, et al. $\left[{ }^{68} \mathrm{Ga}\right]$-albumin-PET in the monitoring of left ventricular function in murine models of ischemic and dilated cardiomyopathy: comparison with cardiac MRI. Mol Imaging Biol. 2013;15:441-449.

27. Porenta G, Kuhle W, Sinha S, et al. Parameter estimation of cardiac geometry by ECG-gated PET imaging: validation using magnetic resonance imaging and echocardiography. J Nucl Med. 1995;36:1123-1129.

28. Stegger L, Heijman E, Schafers KP, Nicolay K, Schafers MA, Strijkers GJ. Quantification of left ventricular volumes and ejection fraction in mice using PET, compared with MRI. J Nucl Med. 2009;50:132-138.

29. Cheng JC, Shoghi K, Laforest R. Quantitative accuracy of MAP reconstruction for dynamic PET imaging in small animals. Med Phys. 2012;39:1029-1041.

30. Skehan SJ, White JF, Evans JW, et al. Mechanism of accumulation of $99 \mathrm{~m} \mathrm{Tc}-$ sulesomab in inflammation. J Nucl Med. 2003;44:11-18.

31. Matsumura Y, Maeda H. A new concept for macromolecular therapeutics in cancer chemotherapy: mechanism of tumoritropic accumulation of proteins and the antitumor agent smancs. Cancer Res. 1986;46:6387-6392.

32. Heneweer C, Holland JP, Divilov V, Carlin S, Lewis JS. Magnitude of enhanced permeability and retention effect in tumors with different phenotypes: ${ }^{89} \mathrm{Zr}$-albumin as a model system. $J$ Nucl Med. 2011;52:625-633.

33. Gibson JG, Evans WA. Clinical studies of the blood volume. I. Clinical application of a method employing the azo dye "Evans blue" and the spectrophotometer. J Clin Invest. 1937;16:301-316.

34. Giger M, Baumgartner HR, Zbinden G. Toxicological effects of Evans blue and Congo red on blood platelets. Agents Actions. 1974;4:173-180. 